\title{
PHOTOSPHERIC NETWORK FROM STUDY OF MANGANESE LINES
}

\author{
G. ELSTE and R. G. TESKE \\ The University of Michigan, Dept. of Astronomy, Ann Arbor, Mich. 48109, U.S.A.
}

(Received 6 December, 1977; in revised form 16 May, 1978)

\begin{abstract}
Area scans with the multi-channel magnetograph at the Kitt Peak McMath Telescope provided simultaneous measures of equivalent widths in two $\mathrm{Mn}$ lines, continuum intensity and longitudinal magnetic field component. Observations were carried out with apertures of $2.5^{\prime \prime} \times 3.5^{\prime \prime}$ arc and $1^{\prime \prime} \times 1$ " arc. For the network elements, which were identified using the magnetic field as tracer, we developed relationships between $I_{\text {Continuum }}, W_{\lambda}, V_{\text {Doppler }}$ and longitudinal field strength. With a minimum of assumptions it is possible to write from these observed relations an expression connecting true temperature perturbation in the line forming layers of the network to true longitudinal field strength. The results provide some constraints upon possible network models.
\end{abstract}

\section{Introduction}

Observations indicated magnetic field concentrations (Harvey, 1976) in the photospheric network, and possibilities for explaining them theoretically were given by Parker (1977). Difficulties in understanding the insufficiently resolved observations are obvious. Our semiempirical approach is aimed at the network structure in quiet regions. As suggested by one of us (G.E.) the equivalent widths of hyperfine structure broadened lines of $\mathrm{Mn} \mathrm{I}$ are safe indicators for temperature, independent of velocity and magnetic fields. We observed with the multi-channel magnetograph on the McMath Telescope at KPNO. Properties of the Mn lines are discussed in Section 2, observations in Section 3, their possible interpretations in terms of heterogeneous photospheres in Section 4, and results in Section 5.

\section{The Manganese Lines and Their Properties}

\section{A. GENERAL CONSIDERATIONS}

Temperature fluctuations in the line forming strata produce equivalent width fluctuations. Hyperfine structure broadening suppresses the influence of non-thermal motions of small dimensions ('microturbulence'). Lines of neutral metals are known to be independent of electron pressure. The Mn I lines $\lambda 5395(\chi=0 \mathrm{eV})$ and $\lambda 5420(\chi=2.1 \mathrm{eV})$ are best suited for our purpose. The nearby Fe $\mathrm{r}$ line $\lambda 5393$ $(\chi=3.2 \mathrm{eV})$ with Landé factor $g_{\text {eff }}=1.5$ was chosen for measuring the magnetic field. By comparing the observed center-to-limb variation of the equivalent widths of our lines with predictions from solar reference models (Elste, 1967; Vernazza et al., 1976) using LTE physics, our lines were tested extensively and found to be reliable temperature indicators in the presence of velocity and magnetic fields. The hyperfine structure splitting and the Zeeman pattern for $2000 \mathrm{G}$ are shown in Figure 1. For the 

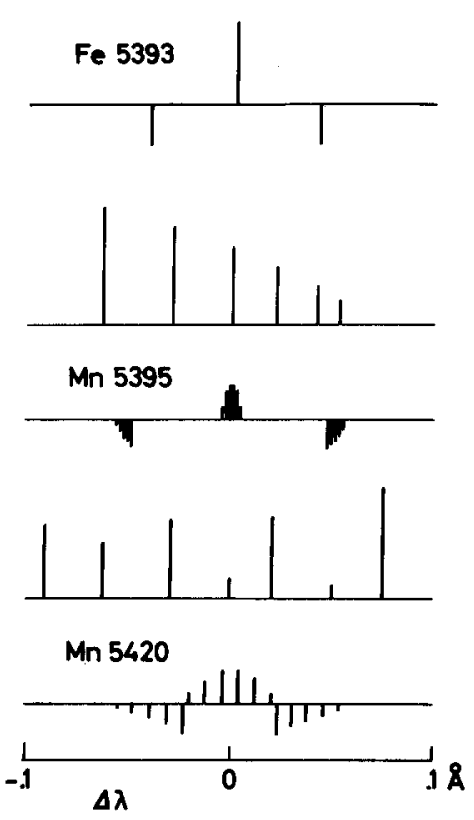

Fig. 1. Zeeman pattern for $2000 \mathrm{G}$ and hyperfine structure splitting for the lines observed.

Mn $\lambda 5395$ line the presence of a longitudinal magnetic field of $2000 \mathrm{G}$ over the entire magnetograph entrance aperture would spread the $\sigma$-components by \pm $50 \mathrm{~m} \AA$, so that a magnetograph exit slit, chosen $340 \mathrm{~m} \AA$ wide, would detect an equivalent width only $0.94 \%$ smaller than that measured without field. Since on few occasions the extent of magnetic regions of the quiet photosphere will cover more than half of the aperture, we conclude that the effect of combined magnetic and hyperfine structure splitting on the measured equivalent width is negligible for the magnetograph exit slit of $340 \mathrm{~m} \AA$ which we used. Similar considerations dictated the choice of a $405 \mathrm{~m} \AA$ exit slit at $\mathrm{Mn} \lambda 5420$ in order to insure negligible effects of magnetic field strength upon the measured $W_{\lambda}$ even in the presence of large velocity shifts.

\section{B. CalCulation of THE TEMPERATURE SENSitivity of THE MANGanese LINES}

To facilitate an analysis of the measured fluctuations of $W_{\lambda}$ for the $\mathrm{Mn}$ lines, we determined from perturbed photospheric models three coefficients $A_{\lambda}, B_{\lambda}, C_{\lambda}$ in expressions of the form

$$
\log \left(W_{\lambda} / \lambda\right)=A_{\lambda}+B_{\lambda}(\log \theta)+C_{\lambda}(\log \theta)^{2},
$$

where $\theta=5040 / T$ refers to the median depth of line formation. We used such expressions to convert observed fluctuations $\Delta W_{\lambda}$ into inferred fluctuations $\Delta \theta$ of inverse temperature in the network. Because we also wished to use our observations 
to investigate the horizontal temperature distribution in the non-network photosphere, the calculations were made for a heterogeneous, multi-element photosphere. Starting with $\theta$ versus $\log \tau_{0}$ stratifications in three columns similar to that in the reference model, two kinds of heterogeneous models were generated imposing the following conditions:

(i) hydrostatic pressure structure in each column,

(ii) horizontal pressure balance in each geometrical depth.

The basic scheme of the model calculations and line calculations is described in the Appendix. The resulting information about the temperature sensitivity of the two manganese lines for the two types of pressure stratification is presented in Table $I$. In that Table, column 3 lists the median optical depths of formation of the line cores; column 4 gives the value of $\theta=5040 / T$ at that median depth. Column 5 lists the

TABLE I

Temperature sensitivity of the two manganese lines

\begin{tabular}{|c|c|c|c|c|c|}
\hline$\theta$-factor & $\log W_{\lambda} / \lambda$ & $\log \tau_{\text {Median }}$ & $\theta$ & Coefficients & $\frac{\mathrm{d} \log W_{\lambda}}{\mathrm{d} \log \theta}$ \\
\hline & Hydrostat & quilibrium & \multicolumn{3}{|c|}{$\frac{\mathrm{d} \log I_{c}}{\mathrm{~d} \log \theta}=-4.17 \quad$ at $\quad \theta=0.73$} \\
\hline & & $\lambda 5395$ & \multicolumn{3}{|c|}{$\log g f A=-10.830$} \\
\hline $\begin{array}{l}0.95 \\
1.00 \\
1.05\end{array}$ & $\begin{array}{l}-5.113 \\
-4.855 \\
-4.674\end{array}$ & $\begin{array}{l}-1.50 \\
-1.67 \\
-1.94\end{array}$ & $\begin{array}{l}0.993 \\
1.063 \\
1.142\end{array}$ & $\begin{array}{l}A=-5.083 \\
B=9.847 \\
C=-47.919\end{array}$ & 7.304 \\
\hline & & $\lambda 5420$ & \multicolumn{3}{|c|}{$\log g f A=-9.480$} \\
\hline $\begin{array}{l}0.95 \\
1.00 \\
1.05\end{array}$ & $\begin{array}{l}-5.018 \\
-4.830 \\
-4.682\end{array}$ & $\begin{array}{l}-1.15 \\
-1.25 \\
-1.36\end{array}$ & $\begin{array}{l}0.952 \\
1.015 \\
1.080\end{array}$ & $\begin{array}{l}A=-4.871 \\
B=6.412 \\
C=-23.068\end{array}$ & 6.113 \\
\hline
\end{tabular}

Horizontal pressure balance $\quad \frac{\mathrm{d} \log I_{c}}{\mathrm{~d} \log \theta}=-4.1$ at $\theta=0.73$

\begin{tabular}{|c|c|c|c|c|c|}
\hline \multirow{4}{*}{$\begin{array}{l}0.93 \\
1.00 \\
1.108\end{array}$} & \multirow{4}{*}{$\begin{array}{l}-5.290 \\
-4.855 \\
-4.522\end{array}$} & \multirow{4}{*}{$\begin{array}{r}\lambda 5395 \\
-1.39 \\
-1.67 \\
-2.26\end{array}$} & \multicolumn{3}{|c|}{$\log g f A=-10.825$} \\
\hline & & & 0.953 & $A=-5.078$ & \\
\hline & & & 1.063 & $B=9.412$ & 7.431 \\
\hline & & & 1.244 & $C=-37.335$ & \\
\hline & \multicolumn{2}{|r|}{$\lambda 5420$} & \multicolumn{2}{|c|}{$\log g f A=-9.469$} & \\
\hline 0.93 & -5.147 & -1.06 & 0.918 & $A=-4.873$ & \\
\hline 1.00 & -4.830 & -1.25 & 1.015 & $B=6.694$ & 6.454 \\
\hline 1.108 & -4.498 & -1.39 & 1.173 & $C=-18.613$ & \\
\hline
\end{tabular}


coefficients $A_{\lambda}, B_{\lambda}, C_{\lambda}$ which were introduced at the beginning of this section. Note that the Table also lists the rate of change of $\log I_{\text {continum }}$ with $\log \theta$. All logarithms are Napierian.

\section{The Observations}

Our need to obtain measures of point-to-point fluctuations of equivalent width free of continuum intensity fluctuations prevented us from using filtroheliograms, which are potentially the data of highest possible angular resolution. In choosing, then, between the use of photographic spectrophotometry or a scanning magnetograph, we were guided by the fact that a magnetograph's angular resolution is not necessarily much worse than that of the finite slit used in spectrophotometry, while the photoelectric magnetograph provides data of considerably better photometric accuracy. We therefore used the multi-channel magnetograph at Kitt Peak (Livingston and Harvey, 1971a) to make simultaneous observations in several wavelengths for intensity, magnetic field and velocity. The observations took the form of raster scans at the main spectrograph of the McMath Telescope.

\section{A. RASTER CONFIGURATIONS}

We started our observing session on 9 May with the frequently used raster aperture of $2.5^{\prime \prime} \times 3.5^{\prime \prime}$ arc, with the $3.5^{\prime \prime}$ dimension in the east-west scan line direction. Samples were taken at positions separated by $1.75^{\prime \prime}$ arc. These were later combined in pairs for effective sample spacings of $3.5^{\prime \prime}$ arc. Completion of a $175^{\prime \prime} \times 175^{\prime \prime}$ raster scan frame took about $22 \mathrm{~min}$. Due to the excellent seeing conditions experienced on the first three days, we decided on May 11 to switch to a $1^{\prime \prime} \times 1$ " aperture. The length of the scan lines was kept the same, $175^{\prime \prime}$ arc, with samples separated by $0.5^{\prime \prime}$ arc, which again were later combined in pairs. Completion of a $175^{\prime \prime} \times 78^{\prime \prime}$ scan frame also took about $22 \mathrm{~min}$.

We expected photospheric oscillations to contribute 'noise' to the network data. This noise source can be suppressed by combining scans made at $150 \mathrm{~s}$ time intervals (Skumanich et al., 1975) or by extensive averaging of scans. To assure ourselves of a scan area large enough to contain several network elements, we elected to use the latter approach.

\section{B. CALIBRATIONS AND NOISE}

Let $\Delta \lambda, S$, and $G$ be the slit width, signal, and gain respectively, with subscripts $\mathrm{Mn}$ and $c$ distinguishing between line and continuum. The equivalent width is then given by

$$
\left(W_{\lambda}\right)_{\mathrm{Mn}}=\left[1-\left(S_{\mathrm{Mn}} / S_{c}\right)\left(G_{c} \Delta \lambda_{c} / G_{\mathrm{Mn}} \Delta \lambda_{\mathrm{Mn}}\right)\right] \Delta \lambda_{\mathrm{Mn}} .
$$

The calibration factor $G_{c} \Delta \lambda_{c} / G_{M n} \Delta \lambda_{M n}$ can be obtained from a wavelength scan, in which each exit slit and detector passes through the spectrum range of interest and at some instant measures the signal of every other wavelength position. Such 
wavelength scans for calibrating equivalent widths as well as standard drift curve calibrations for velocity and magnetic field measurements were obtained daily. Resulting equivalent widths agreed well with those measured directly on atlas tracings. Individual velocity and magnetic field factors agreed within 3 and 5\% respectively. True instrumental noise in all channels was determined with a defocussed telescope and an integrating lens for both aperture size arrangements. The resulting RMS noise levels are listed in Table II.

\section{AVERAGE BACKGROUND DETERMINATION}

We seek to measure and to correlate fluctuations of intensity, velocity, equivalent width and magnetic field within the scan frames. With the exception of the velocity the fluctuations will always be defined as the difference local value minus average expressed in units of the average. The determination of the average is essential. We expect that the average of solar photospheric conditions will be faithfully represented in raster frames which do not contain active regions. Our attention was carefully confined to such areas on the Sun, and proof obtained from K-Line spectroheliograms.

It appeared that data along scan lines could be well fitted by parabolas of small curvature; parabolas also fitted well to strings of data across scan lines. Moreover, model atmosphere calculations for continuum intensity and for equivalent width of the $\mathrm{Mn}$ lines showed that at all limb distances $\cos \theta \geq 0.5$ the radial distribution of quantities on a $175^{\prime \prime}$ arc scan line could be fitted to within $0.1 \%$ by a parabola.

We think of the data as consisting of a mixture of two properties: a random, noise-like distribution associated with the granulation and $5 \mathrm{~min}$ oscillations, and a non-random tail superposed on that distribution which represents the network. A single least-squares fit of a parabolic surface to the data will yield a gaussian distribution of residuals and may mask the network. Therefore we fit a second surface to the data: All data points which lie more than one standard deviation from the first surface are cast out, and a second parabolic surface fitted to the remaining points. The resulting surface is then assumed to represent the mean background to which all fluctuations are referred.

D. REMOVAL OF STREAKS

In fitting parabolas to scan lines obtained with the $2.5^{\prime \prime} \times 3.5^{\prime \prime}$ aperture we found a persistent pattern of streaks in the data. These have been studied and removed from all scans.

\section{Global Properties of the Heterogeneous Photosphere}

\section{A. ROOT MEAN SQUARE FLUCTUATIONS}

The RMS fluctuations of our measurements are listed in columns 4-7 of Table II. For simplicity we just mark the proper dispersions and correlation coefficients of the 


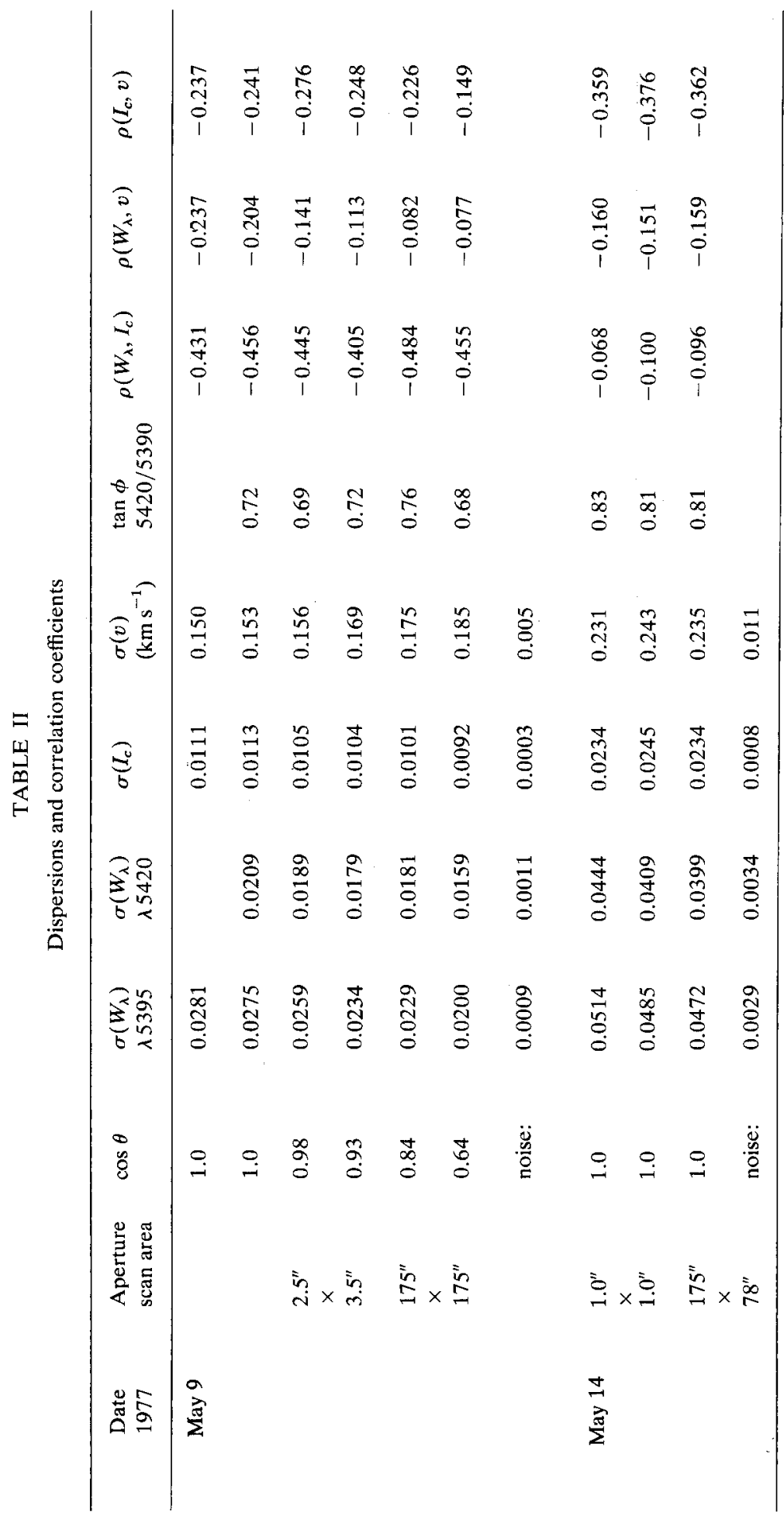


fluctuations by the symbol to which they apply. Fortunately we have data for two aperture sizes which are very little falsified by noise. The values for the continuum intensity behave as expected for the unresolved granulation structure observed with $1^{\prime \prime}$ arc seeing disk, comparing well with the value 0.050 measured by Deubner and Mattig (1975).

Center-to-limb variations were only observed by us with the large $\left(2.5^{\prime \prime} \times 3.5^{\prime \prime}\right)$ probe. We find a decrease of the RMS intensity fluctuations with increasing distance from disk center (Table II), while high resolution Stratoscope observations showed increasing contrast (Edmonds, 1962). This is no contradiction, but means that the fine structure is lost in the extensive averaging by our large probe, while fluctuations over large areas actually decrease.

For the velocities the observed growth of the RMS fluctuations with distance from disk center (large probe only) confirms the presence of faster horizontal motions in the photosphere found previously, as summarized by Canfield and Beckers (1975). Also the value of our RMS velocity fluctuations agrees very well with data derived from slit spectra of high spatial resolution (Beckers and Canfield, 1975).

Since continuum intensity and velocity fluctuations compare well with those found by previous investigations, we may trust the RMS fluctuations in the equivalent width of the manganese lines, and estimate the actual fluctuations at high resolution to be up to twice as large as directly measured by us. The influence of resolution on the correlation of observed quantities will be discussed in a separate paper.

\section{B. CORRELATIONS BETWEEN EQUIVALENT WIDTH FLUCTUATIONS}

An example of a plot relating the fluctuations $\Delta W_{\lambda} /\left\langle W_{\lambda}\right\rangle$ in the $\operatorname{Mn} \lambda 5395$ and $\mathrm{Mn}$ $\lambda 5420$ lines at $1^{\prime \prime} \times 1^{\prime \prime}$ resolution is shown in Figure 2 . The considerable scatter in the

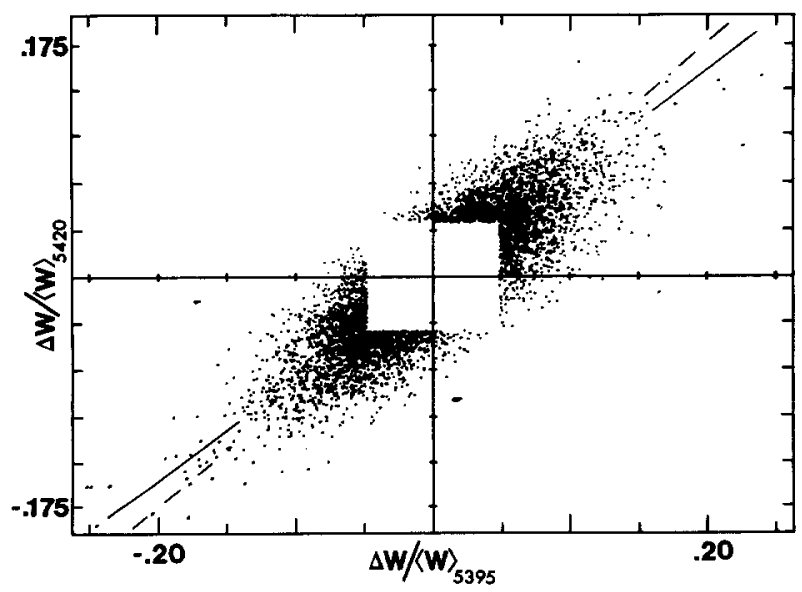

Fig. 2. Scatter diagram for equivalent width fluctuations in $\lambda 5420$ as a function of those in $\lambda 5395$ for a $1^{\prime \prime} \times 1^{\prime \prime}$ raster of 14 May. The solid line indicates the prediction for hydrostatic equilibrium, the dashed line that for horizontal pressure balance. 
data is intrinsically photospheric. The general trend is due to the different temperature sensitivities of the two lines while the scatter is due to local variations of the photospheric structure. Standard deviations for the equivalent width fluctuations are listed in Table II. In column 8 we give the slope of a linear relation between the equivalent width fluctuations in the two lines taking into account that both measures scatter intrinsically.

We now recall that our simple model calculations showed a dependence of the temperature sensitivity of the two manganese lines on the assumed pressure structure (see Table I). Calculating the gradient $\left[\mathrm{d} \log W_{\lambda} / \lambda(5420)\right] /\left[\mathrm{d} \log W_{\lambda} / \lambda(5395)\right]$ from column 2 of Table I leads to 0.78 for hydrostatic equilibrium in each column. The corresponding relations are indicated in Figure 2. The average observed gradient is 0.71 for the large size probe and 0.81 for the small sized probe. We expect that observations of the solar atmosphere made at low resolution would tend to give results as if one were looking at an atmosphere in hydrostatic equilibrium while, as resolution improves and a few single structural elements are isolated, the observed radiation field would tend towards that for the horizontal pressure balance case. This expectation appears to be borne out by the present data.

\section{CORRELATIONS BETWEEN CONTINUUM BRIGHTNESS AND EQUIVALENT WIDTH}

We have found, in agreement with many others, that continuum brightness correlates poorly with velocity and with magnetic field. This is also true for equivalent width of the Mn $\lambda 5395$ line (see Table II). Data for a typical raster are shown in Figure 3 . The low correlations have been explained as being caused by phase lag effects in photospheric oscillations (Evans and Michard, 1962; Tanenbaum et al., 1969; Mehltretter, 1971; Holweger and Testerman, 1975). We note, however, that Edmonds and Webb (1972) investigated continuum intensity and equivalent width

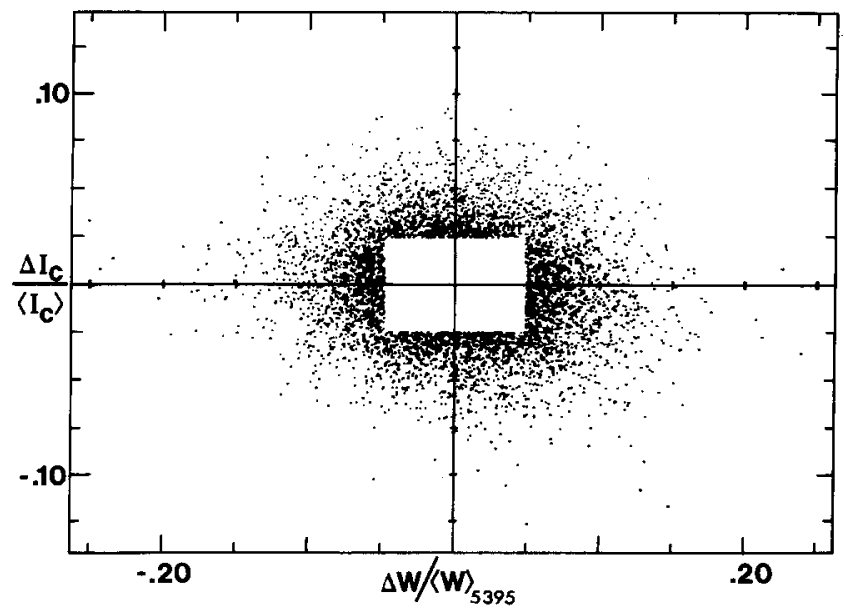

Fig. 3. Scatter diagram for continuum intensity fluctuations as a function of equivalent width fluctuations in $\lambda 5395$ for the same data set as in Figure 2. 
fluctuations in the highly temperature sensitive $C_{\text {I }} \lambda 5052$ line, which is formed in even deeper layers than the Mn lines, and also found a lack of correlation. Their study of the phase of various fluctuations was inconclusive.

While continuum intensities are determined by the local temperature, the equivalent widths of lines are dependent upon the temperature gradient as well as upon the local temperature. Since our atmosphere models only involved scaled temperature distributions they predict a high correlation of continuum brightness and line equivalent width. The low correlations found by us and by many workers point strongly towards variations of temperature gradient in the photospheric inhomogeneities. This, of course, would produce a noise-like behavior of quantities pertaining to different strata. Another complication in the interpretation of correlations arises from the well known observational fact, that the continuum brightness in the center of granulae of the same size varies, probably randomly.

\section{The Network}

\section{A. IDENTIFICATION OF NETWORK AREAS}

The network field pattern was located by us following an approach used by Skumanich et al. (1975). We conceive, with them, of a distribution of magnetic fields on the photosphere which contains a random, noise-like background on which are superposed the network fields. This process described in Section 3.C, applied to the magnetic field data, gave a distribution of magnetic fields which had a symmetric gaussian core and extended wings which we have assumed to be of network origin. Following Skumanich et al. (1975) we fitted to the symmetric central peaks gaussian distributions of the form ( $N=$ the number of non-network picture elements)

$$
y=\frac{N}{\sigma_{0} \sqrt{2 \pi}} \exp \left[-\left(H-H_{0}\right)^{2} / 2 \sigma_{0}^{2}\right]
$$

using a differential corrections method.

The dispersion of the background fields corrected for system noise is found to increase with resolution from $4.5 \mathrm{G}\left(2.5^{\prime \prime} \times 3.5^{\prime \prime} \mathrm{arc}\right)$ to $8.6 \mathrm{G}\left(1^{\prime \prime} \times 1^{\prime \prime} \mathrm{arc}\right)$. The low resolution values are consistent with those quoted by Livingston and Harvey (1971b) ( 2 to $3 \mathrm{G}$ at $5^{\prime \prime}$ arc resolution). Skumanich et al. (1975) with $1.1 \pm 0.6 \mathrm{G}$ dispersion at $2.4^{\prime \prime} \times 2.4^{\prime \prime}$ arc may have overestimated their system noise of $3.0 \mathrm{G}$, although the values are not directly comparable since they were obtained with different spectrum lines.

A subtraction from the magnetic field distribution of the gaussian which best fits the noise-like core yields a network cut-off value of $\pm 10 \mathrm{G}$ at $2.5^{\prime \prime} \times 3.5^{\prime \prime}$ arc and $\pm 30 \mathrm{G}$ at $1^{\prime \prime} \times 1 "$ arc. We do not think that the observed fractional area of the network is influenced by resolution so long as this fraction is obtained by the procedure we have borrowed. Rather, $\left(1-N / N_{\text {total }}\right)$ will be principally determined by the physical conditions in the scan area. Skumanich et al. (1975) found the 
fractional network area to be 0.38 for a quiet area near disk center of 1968.8. Our data refer to particularly quiet areas on the photosphere, and, with cut-off values like that used by Skumanich et al. (1975) give a network fraction of from 0.09 to 0.18 at disk center. Both our high and low resolution data yield an approximate density of network fragments of $3 \times 10^{-3} \mathrm{Mm}^{-2}$, comparable to the $2 \times 10^{-3} \mathrm{Mm}^{-2}$ derived by Skumanich et al. (1975) despite the smaller overall area of the fragments themselves. We have compared our network locations with the $\mathrm{CN}$ and $\mathrm{Ca}$ II spectroheliograms made at the time of the scans, finding an almost perfect spatial relationship between the network contours and bright areas on the spectroheliograms. This also shows us that we have selected really quiet regions sufficiently separated from any plage. Using $\pm 30 \mathrm{G}$ cutoff, $9 \%$ of the area is occupied by the network. This is much less than the fraction derived by Skumanich et al. (1975) and is probably due to their having inadvertently included in their scan area a non-typical region. Ca II spectroheliograms made at McMath-Hulbert Observatory show weak plage material spreading to the disk center at the time of their observations.

Tanenbaum et al. (1971) have found magnetic 5 min oscillations of amplitude 1 to $2 \mathrm{G}$; it is clear, however, that the effect of such variations will be small in locating the network.

\section{B. MANGANESE EQUIVALENT WIDTH FLUCTUATIONS IN THE NETWORK}

Expecting that temperature fluctuations associated with magnetic fields ought to be independent of the sense of those fields (Chapman and Sheeley, 1968; Frazier, 1970), we have studied the relations between line equivalent width and absolute value of field. In Figure 4 is shown a scatter plot of $\Delta W_{5395} /\left\langle W_{5395}\right\rangle$ versus $\left|H_{\|}\right|$for one high resolution raster. The equivalent widths for this line and for the $\mathrm{Mn} \lambda 5420$ line decrease strongly in network fields. For fields stronger than the cut-off value of $\pm 30 \mathrm{G}$ the average linear regressions of equivalent width fluctuations with absolute field strength are listed in Table III. The 5 min oscillations contribute some noise to

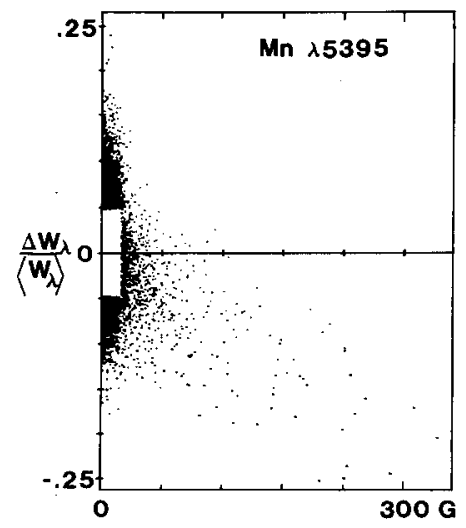

Fig. 4. Equivalent width fluctuations in $\lambda 5395$ as a function of absolute magnetic field strength for the $1^{\prime \prime} \times 1$ " arc raster of 14 May. 
these data but, from the work by Holweger and Testerman (1975), we find it is negligible.

\section{CONTINUUM INTENSITY FLUCTUATIONS IN THE NETWORK ELEMENTS}

Continuum brightness fluctuations due to granulation and associated with the 5 min oscillations can effectively mask the brightness associated with the network (cf. Skumanich et al., 1975). Using the same procedure as for the equivalent widths we obtained the averaged linear relations for the continuum given in Table III. The slope for low resolution data agrees well with Skumanich et al. (1975): $9.3 \times$ $10^{-5} \mathrm{G}^{-1}$. That they derived a higher correlation coefficient $(0.23)$ than did we $(0.16 \pm 0.07)$ is indicative of the rejection of continuum brightness oscillations by their observing technique.

TABLE III

Linear regression relations for photospheric network $Y=\beta+\gamma \cdot\left|H_{0}^{\prime}\right|$ with $\left|H_{0}^{\prime}\right|$ in units of $10^{3} \mathrm{G}$

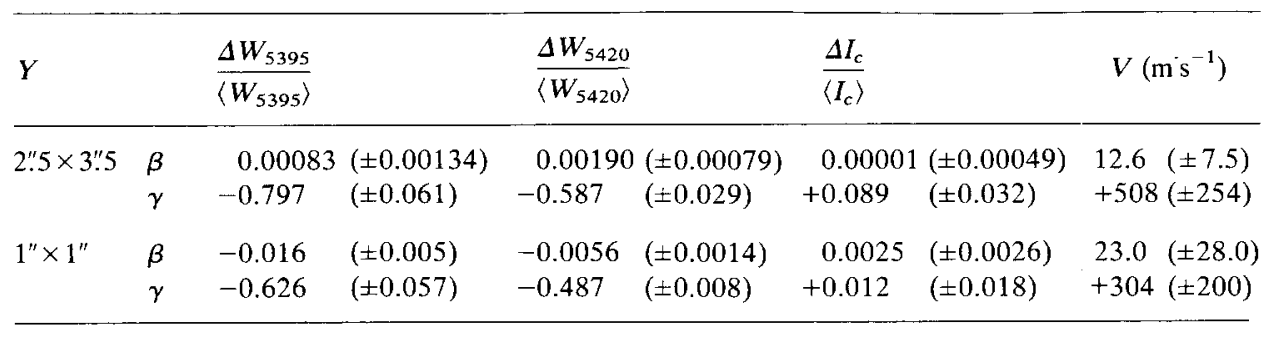

The agreement of slopes suggests that the scales of magnetic field measures used by them and by us are in accord. We also note that the maximum field values of $\sim 200 \mathrm{G}$ obtained by us at $2.5^{\prime \prime} \times 3.5^{\prime \prime}$ aperture are comparable to their maximum field values of $\sim 180 \mathrm{G}$.

\section{VELOCITIES IN THE NETWORK ELEMENTS}

The observed velocity signal is strongly controlled by oscillatory effects and is the most poorly determined quantity in our present series of observations. In Table III we have listed the average intercepts and slopes of the linear regressions linking velocity and absolute vertical field strength for network elements. The sense of these velocities is: positive $=$ receding from observer $=$ downdraft .

\section{E. APPROXIMATION TO THE NETWORK STRUCTURE}

The relations of Table III can be used to provide some coarse information about the temperature and pressure perturbations in network elements independently of their resolution. Current evidence supports the view that the network consists ultimately of individual magnetic elements or flux tubes (e.g. the review by Harvey, 1976) which 
are of small horizontal size. For the flux tubes we will assume characteristic mean values of: cross-section, field strength, temperature structure and gas flow speed.

Let a flux-tube's cross-sectional area be $a$ in $(\operatorname{arcsec})^{2}$ and the magnetograph's entrance aperture $A$ in the same units. Any given picture element then contains a fractional area $a / A$ of flux tubes. The observed value of an equivalent width $W_{\lambda}^{\prime \prime}$, will therefore depend upon the mean inter-network value $\left\langle W_{\lambda}\right\rangle$, upon the true perturbed value $W_{\lambda}^{\prime}$ in the network and upon the fractional aperture $a_{\lambda} / A$ covered by network elements:

$$
W_{\lambda}^{\prime \prime}=\left(1-\frac{a_{\lambda}}{A}\right)\left\langle W_{\lambda}\right\rangle+\frac{a_{\lambda}}{A} W_{\lambda}^{\prime}
$$

Our observations have provided the dependence of $W_{\lambda}^{\prime \prime}$ upon measured absolute field strength $\left|H_{0}^{\prime \prime}\right|$ (see Table III):

$$
W_{\lambda}^{\prime \prime}=\left\langle W_{\lambda}\right\rangle+\left\langle W_{\lambda}\right\rangle\left[\beta_{\lambda}+\gamma_{\lambda}\left|H_{0}^{\prime \prime}\right|\right]
$$

In this initial survey we will assume that the intercepts $\beta_{\lambda}$ of the regression lines are truly zero and that errors have led to non-zero values in the table.

The values of the observed magnetic field $H_{0}^{\prime \prime}$ will depend on the fractional area $a_{H} / A$ occupied by the magnetic elements with a field strength $H^{\prime}$, and on the strength of an average background field $H_{B}^{\prime}$ which may exist in the immediate surrounding of the magnetic elements:

$$
H_{0}^{\prime \prime}=\left(1-\frac{a_{H}}{A}\right) H_{B}^{\prime}+\frac{a_{H}}{A} H^{\prime}
$$

where we may leave it open if the weak background field has the same or opposite polarity as the network field.

Combining Equations (4) and (5) we obtain, for $\beta_{\lambda}=0$,

$$
\frac{a_{\lambda}}{A} \frac{\Delta W_{\lambda}^{\prime}}{\left\langle W_{\lambda}\right\rangle}=\gamma_{\lambda}(A)\left|H_{0}^{\prime \prime}\right|
$$

Since we don't know the strength and polarity of the background fields we may set them to zero and first approximate $\left|H_{0}^{\prime \prime}\right|$ by $\left(a_{H} / A\right)\left|H^{\prime}\right|$. This leads to a relation between the true equivalent width fluctuations and the true fields in the network, in which the direct dependence on the magnetograph aperture $A$ has vanished,

$$
\frac{\Delta W_{\lambda}^{\prime}}{\left\langle W_{\lambda}\right\rangle}=\frac{a_{H}}{a_{\lambda}} \gamma_{\lambda}(A)\left|H^{\prime}\right|,
$$

while an indirect dependence on aperture area $A$ still exists through the factors $\gamma_{\lambda}(A)$ (Table III). 
The link between the true equivalent width fluctuations in each manganese line and the true temperature fluctuations is provided by our model atmosphere calculations. From Equation (1) we obtain

$$
\mathrm{d}\left(\ln W_{\lambda}\right)=\left(B_{\lambda}+2 C_{\lambda} \log \theta_{\lambda}\right) \frac{\mathrm{d} \theta}{\theta_{\lambda}}
$$

where $\theta_{\lambda}$ takes the value at the median depth of line formation and the coefficients $B_{\lambda}, C_{\lambda}$ are in Table I. Inserting this into Equation (8) we have

$$
\frac{B_{\lambda}+2 C_{\lambda} \log \theta_{\lambda}}{\theta_{\lambda}} \Delta \theta=\frac{a_{H}}{a_{\lambda}} \gamma_{\lambda}\left|H^{\prime}\right| .
$$

There are four such equations, one for each line at each of two resolutions.

Under the assumption that $\Delta \theta$ is the same in the line-forming layers for both lines, we obtain from (10) diameter ratios $a_{\lambda 5395} / a_{\lambda 5420}$ with values $1.13 \pm 0.12$ and $1.19 \pm 0.16$ for high and low resolution respectively. A conclusion that network elements are slightly wider at the $\lambda 5395$ line level of the atmosphere than at the $\lambda 5420$ line level is thus marginally indicated. Because the lines used in our work are formed at so nearly the same atmospheric altitude (only $60 \mathrm{~km}$ apart, Table IV) we question whether an effect on the scale of Chapman's (1977) model could be detectable with the present observations.

TABLE IV

\begin{tabular}{lllll}
\hline & & & \multicolumn{2}{c}{$\Delta \theta /\left(\frac{a_{H}}{a_{\lambda}}\left|H^{\prime}\right|\right) \mathrm{in} \mathrm{deg}^{-1}\left(10^{5} \mathrm{G}\right)^{-1}$} \\
\cline { 3 - 5 } $\begin{array}{l}\text { Spectrum } \\
\text { feature }\end{array}$ & $\log \tau_{5000}$ & $\Delta h(\mathrm{~km})$ & $2.5^{\prime \prime} \times 3.5^{\prime \prime}$ & $1^{\prime \prime} \times 1^{\prime \prime}$ \\
\hline Mn $\lambda 5395$ & -1.67 & 244 & $11.6 \pm 0.9$ & $9.1 \pm 0.8$ \\
Mn $\lambda 5420$ & -1.25 & 184 & $9.7 \pm 0.5$ & $8.1 \pm 0.1$ \\
Continuum & +0.2 & 0 & $1.6 \pm 0.6$ & $0.2 \pm 0.3$ \\
\hline
\end{tabular}

With Equation (10) and the data in Tables I and III we have calculated the coefficients

$$
\Delta \theta /\left(\frac{a_{H}}{a_{\lambda}}\left|H^{\prime}\right|\right)
$$

in units of $\operatorname{deg}^{-1}\left(10^{5} \mathrm{G}\right)^{-1}$, given in Table IV. If we knew the field strength in the network and the ratio of diameters of magnetic and thermal perturbations, a mean $\Delta \theta$ could be calculated which referred to the levels of line and continuum formation. Such information is a very useful constraint upon possible network atmospheric models.

Because our calculation of $a_{\lambda 5395} / a_{\lambda 5420}$ above gave a marginal result of 1.0 , we now adopt the view that a network element is cylindrical, of constant radius and with 
a uniform field strength at all observable depths. Under these assumptions our measurements indicate that $\Delta \theta$ is largest at the level of formation of $\mathrm{Mn} \lambda 5395$ and smallest, perhaps even zero, at the level of continuum formation. Hence the temperature gradient in a network element, expressed in terms of the photospheric depth scale, may be much flatter than the photospheric gradient. That is, the above assumptions lead to a general temperature structure like that of current facular (network) models.

This should not be taken to imply that we repudiate the basis for the inhomogeneous solar photospheric model 10, which was introduced in Section 2 as a description of the heterogeneous photosphere and in which $\Delta \theta$ is constant with depth in hot and cold columns. Rather, the present work suggests that $\Delta \theta$ depends upon depth in network elements.

On the other hand, the coefficients in Table IV do not support Chapman's facular (network) model (1977). Employing those coefficients together with $\Delta \theta$ from his model $7 \mathrm{~B} 13 / \mathrm{HRSA}$ and $H^{\prime}=1500 \mathrm{G}$ yields values of $a_{\lambda} / a_{H}$ which portray an upwards narrowing of network elements between the two line forming layers. As we pointed out earlier, the assumption of increasing diameter upwards requires a nearly constant $\Delta \theta$, unlike Chapman's model.

We do, however, believe that the present data should be interpreted in terms of a changed gradient of temperature vs $\log \tau_{5000}$ in network elements. With our prior results we may derive an approximate upper limit to the perturbed temperature gradient by assuming that in those picture elements where $H^{\prime \prime}$ is observed to be a maximum, network flux tubes would just fill the $1^{\prime \prime} \times 1^{\prime \prime}$ entrance aperture. With the observed maximum field of $\sim 320 \mathrm{G}$ (e.g. Figure 4 ) and a postulated 1 " arc seeing, the minimum possible network field is about $450 \mathrm{G}$. Using the coefficients of Table IV, assuming $a_{H}=a_{\lambda}$, and with the information in Table I we find that $\Delta T \sim 180 \mathrm{~K}$ in the line-forming layers of the network while $\Delta T$ is very small at the level where the continuum is produced. Again with data from Table I we compute a perturbed temperature gradient of $1060 \mathrm{~K} /(\Delta \log \tau=1)$ between the line-forming and continuum levels as compared with the unperturbed gradient of $1290 \mathrm{~K} /(\Delta \log \tau=$ $1)$. But it is likely that the perturbed gradient is even flatter than calculated here since we have likely used a minimum field value in getting it.

\section{Summary}

Simultaneous measurements of continuum intensity, equivalent width in two $\mathrm{Mn}$ lines, Doppler velocity and longitudinal magnetic field were made in picture elements of quiet area scans using the multi-channel magnetograph at the McMath Telescope. Correlations of equivalent width fluctuations in the two Mn lines indicate that on the quiet photosphere the inhomogeneities preserve a horizontal pressure balance at all depths. Variations of the vertical temperature gradient in the inhomogeneities cause a low correlation between continuum brightness and equivalent width of the Mn lines. 
Network structures were identified using the magnetic field as a tracer. At disk center the fractional area occupied by the network ranged from 0.09 to 0.18 , i.e. less than $50 \%$ of the value derived by Skumanich et al. (1975). Continuum intensity, equivalent width of $\mathrm{Mn}$ lines and velocity are all correlated with longitudinal field in network elements. Relationships between these quantities are somewhat uncertain because of noise contributions by photospheric oscillations. However, that noise source has negligible influence upon the Mn equivalent widths; their relation to the longitudinal field is well established by the data. With them we developed in Equation (10) the relationship between true temperature increase in the lineforming layers and true network magnetic field strength with a minimum of assumptions. The equation incorporates the horizontal dimension of network elements. The results require either (i) elements of nearly uniform cross-section in which the vertical temperature gradient is much flatter than in the photosphere or (ii) elements in which the cross-section grows with altitude but for which the vertical temperature gradient is like the photosphere's. We prefer the former viewpoint and calculate that the temperature gradient per unit $\log \tau$ in network elements is less than $1060 \mathrm{~K}$.

\section{Appendix A: Basic Scheme of Model Calculations}

Our scheme for calculating hydrostatic equilibrium models for a given $\theta$ stratification has been explained in detail by Elste (1968). Depth-independent scale factors were applied to the $\boldsymbol{\theta}$-values to obtain hotter and colder stratifications.

In contrast to the scaled models with hydrostatic equilibrium in each column the horizontal pressure balance model requires further explanation. This heterogeneous atmosphere was developed to explain observations which otherwise cannot be understood. The areal distribution of $25 \%$ in each of the hot and cold regions resembles the appearance of the solar granulation with its narrow dark intergranular lanes quite well. Requiring that the total thermal energy in each geometrical depth equals that of the well established homogeneous atmosphere couples the temperatures in the hot and cold columns and is expressed by

$$
0.25 T_{H}^{4}+0.25 T_{C}^{4}+0.5 T_{M}^{4}=T_{M}^{4}
$$

It was first introduced by Böhm (1954). Choosing $\theta_{H} / \theta_{M}=0.93$ at each geometrical depth led to a fairly good fit of the observed (Edmonds, 1962) RMS intensity fluctuations at disk center as well as their increase with the angle of incidence into the atmosphere up to about $50^{\circ}$. At this angle or even before, the penetration of the observed radiation through several columns of different temperatures must be taken into account, as preliminary calculations have shown. Other observations, explained by this heterogeneous atmosphere, are the shape of the profiles of iron lines (Elste, 1967), and the center-to-limb variation of the asymmetry and line center wavelengths of the infrared oxygen triplet. 


\section{Appendix B: Line Calculations}

The calculations of equivalent widths for our spectrum lines were always obtained by integrating over line profiles. Since we are dealing with relatively weak lines with no chromospheric contributions the calculation of line depths followed the standard approach of local thermodynamic equilibrium. The computer program contains an option to account for radiative ionizations from metastable levels due to the relevant ultraviolet radiation fields important in the cool and narrow intergranular lanes and in the higher layers. The depth dependent line broadening takes into account the thermal motions, (constant) microturbulence, van der Waals broadening and quadratic Stark effect. In addition the program is versatile to also accommodate Zeeman components and/or hyperfine structure splitting. The interaction constants for van der Waals broadening were calculated according to the hydrogenic approximation given by Unsöld (1955), but with an enhancement factor which can be based on recent laboratory experiments and detailed calculations as reported by van Regemorter (1973). The interaction constants for the much less important quadratic Stark effect were estimated from laboratory measurements.

\section{Appendix C: Depths of Line Formation}

The observed profile of the Fe I line $\lambda 5395$ at disk center is well represented by a synthetic profile using the inhomogeneous model 10 with the ionization of iron due to the radiation field of $\lambda 2500 \AA$. The microturbulence was $1.0 \mathrm{~km} \mathrm{~s}^{-1}$ and the two component macroturbulence was $\pm 1.5 \mathrm{~km} \mathrm{~s}^{-1}$. In order to obtain information about the depth of line formation we restrict our consideration to the profile from the stationary medium column. In Figure 5 this profile is compared with the observed

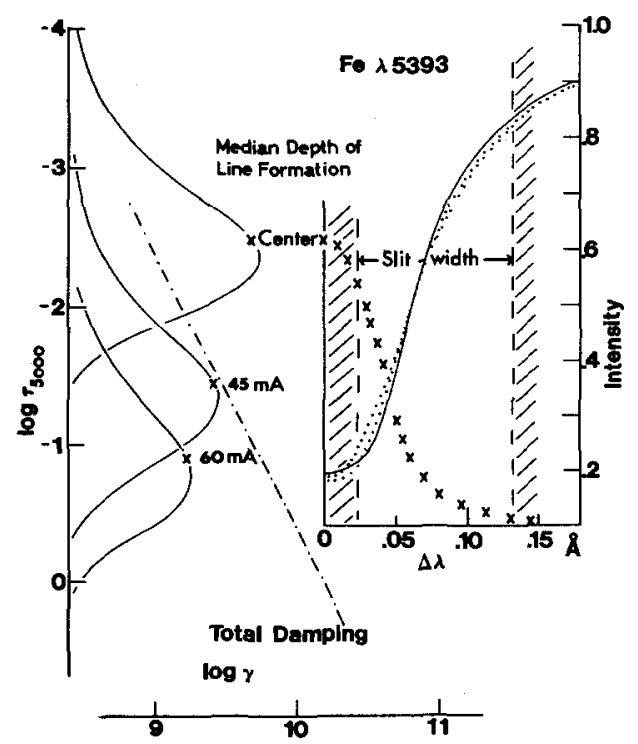

Fig. 5. Slit configuration, line profile, and depth of formation for $\mathrm{Fe} \lambda 5393$. 
asymmetrical profile, as indicated by the dotted curve, which is narrower near the core and wider in the wings. On the left hand side of this figure, we have plotted the contribution to the line absorption as a function of $\log \tau_{5000}$ for three points in the line: line center, $\Delta \lambda=45 \mathrm{~m} \AA$, and $\Delta \lambda=60 \mathrm{~m} \AA$. The median depth values for these curves are marked by $\times$. Superimposed on the line profile the $\times$ 's indicate the values of the median depth of line formation for each distance from the line center. Within the width of the slit we find values of the median depth in the wide range between -2.2 and -0.4 in $\log \tau_{5000}$. It is worth noting that the continuum emission, not shown here, is formed in deeper strata at $\log \tau=+0.2$. Since the damping plays an important role in the formation of this line, the strong depth dependence of the total damping in circular frequencies is indicated as a dashed curve. Next, we consider the situation for the hyperfine structure broadened manganese lines. Figure 6 shows the flat bottom profile observed for the $0 \mathrm{eV}$ line $\lambda$ 5395. The dependence of the median depth of line formation as a function of wavelength is again indicated by $\times$. The

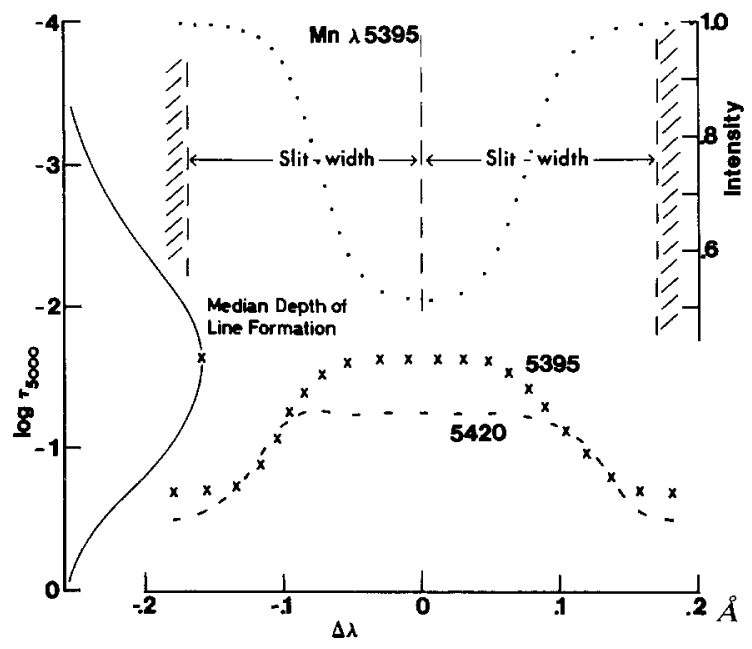

Fig. 6. Slit configuration, line profile, and depth of formation for Mn $\lambda 5395$ and $\lambda 5420$.

corresponding behavior for $\lambda 5420$ is added as a dashed curve. For the purpose of measuring equivalent widths the slits for both manganese lines were chosen much wider than in the case of the iron line. We see that for the Mn 5395 line the median contribution to the depth of line formation comes mainly from layers around -1.6 corresponding to the broad bottom, but extending down to -0.7 in the flanks. The $2.15 \mathrm{eV}$ line $\lambda 5420$ is formed slightly deeper, around -1.2 and extending to -0.5 . We summarize that the strata of line formation of the manganese lines lies definitely deeper than the temperature minimum and above the origin of the continuum. These properties of our lines have to be kept in mind for the interpretation of the measurements. 


\section{Acknowledgements}

We especially thank Dr J. W. Harvey and Mr B. Gillespie for their indispensable assistance in making the observations discussed in this paper. Discussions of calibration techniques with Dr Harvey were helpful.

\section{References}

Beckers, J. M. and Canfield, R. C.: 1975, in R. Cayrel and M. Steinberg (eds.), 'Physique des mouvements dans les atmosphères stellaires', CNRS Colloq. 250, 207.

Böhm, K. H.: 1954, Z. Astrophys. 35, 179.

Canfield, R. C. and Beckers, J. M.: 1975, in R. Cayrel and M. Steinberg (eds.), 'Physique des mouvements dans les atmosphères stellaires', CNRS Colloq. 250, 291.

Chapman, G. A.: 1977, Astrophys. J. Suppl. Ser. 33, 35.

Chapman, G. A. and Sheeley, N. R.: 1968, Solar Phys. 5, 442.

Deubner, F.-L. and Mattig, W.: 1975, Astron. Astrophys. 45, 167.

Edmonds, F. N., Jr.: 1962, Astrophys. J. Suppl. Ser. 6, 357.

Edmonds, F. N., Jr. and Webb, C. J.: 1972, Solar Phys. 25, 44.

Elste, G.: 1967, Astrophys. J. 148, 857.

Elste, G.: 1968, Solar Phys. 3, 106.

Elste, G.: 1972, Bull. Am. Astron. Soc. 4, 424.

Evans, J. W. and Michard, R.: 1962, Astrophys. J. 135, 812.

Frazier, E. N.: 1970, Solar Phys. 14, 89.

Harvey, J.: 1976, in E. A. Müller (ed.), Highlights in Astronomy 4, Part 2, D. Reidel Publ. Co., Dordrecht, Holland, p. 223.

Holweger, H. and Testerman, L.: 1975, Solar Phys. 43, 271.

Livingston, W. C. and Harvey, J.: 1971a, Kitt Peak Nat. Obs. Contr. 558.

Livingston, W. C. and Harvey, J.: 1971b, in R. Howard (ed.), 'Solar Magnetic Fields', IAU Symp. 43, 51.

Mehltretter, J. P.: 1971, Solar Phys. 16, 253.

Parker, E. N.: 1977, Astrophys. J. 210, 816.

Skumanich, A., Smythe, C., and Frazier, E. N.: 1975, Astrophys. J. 200, 747.

Tanenbaum, A. S., Wilcox, J. M., and Howard, R.: 1971, in R. Howard (ed.), 'Solar Magnetic Fields', IAU Symp. 43, 348.

Tanenbaum, A. S., Wilcox, J. M., Frazier, E. N., and Howard, R.: 1969, Solar Phys. 9, 328.

Unsöld, A.: 1955, Physik der Sternatmosphären, 2nd ed., Springer Berlin, Ch. XI.

Vernazza, J. E., Avrett, E. H., and Loeser, R.: 1976, Astrophys. J. Suppl. Ser. 30, 1.

Van Regemorter, J.: 1973, in C. de Jager (ed.), IAU Reports on Astronomy XVA, 160. 